\title{
HAND
}

\section{A systematic review of treatment Interventions for metacarpal shaft fractures in adults}

\begin{tabular}{|c|l|}
\hline Journal: & HAND \\
\hline Manuscript ID & Draft \\
\hline Keywuscript Type: & Review Articles \\
\hline Keyws: & $\begin{array}{l}\text { Fracture/dislocation < Diagnosis, Finger < Fracture/dislocation < } \\
\text { Diagnosis, Trauma < Diagnosis, Treatment < Research \& health } \\
\text { outcomes, Evaluation < Research \& health outcomes }\end{array}$ \\
\hline $\begin{array}{l}\text { Abstract: } \\
\text { predominantly affect younger patients. There is wide variability in their } \\
\text { treatment with no consensus on best practice. We performed a } \\
\text { systematic review to assess the breadth and quality of available evidence } \\
\text { supporting different treatment modalities for metacarpal shaft fractures } \\
\text { of the finger digits in adults. A comprehensive search was conducted } \\
\text { across multiple databases, in line with Preferred Reporting Items for } \\
\text { Systematic Reviews and Meta-Analyses (PRISMA) guidelines. A total of } \\
\text { 1600 records were identified; seven studies fulfilled eligibility criteria and } \\
\text { were included. No randomised controlled trials directly comparing } \\
\text { surgery to non-surgical treatment were found. One retrospective study } \\
\text { compared non-surgical to surgical treatment, while six compared surgical } \\
\text { or non-surgical treatments. Considerable heterogeneity between studies } \\
\text { along with high or critical risk of bias restricts direct comparison and } \\
\text { conclusions. There is a lack of high quality evidence to guide treatment, } \\
\text { supporting the need for well-designed, multi-centre trials to identify the } \\
\text { most effective and cost-efficient treatment for metacarpal shaft fractures } \\
\text { in adults. }\end{array}$ \\
\hline
\end{tabular}

\section{SCHOLARONE" Manuscripts}




\title{
A SYSTEMATIC REVIEW OF TREATMENT INTERVENTIONS FOR METACARPAL SHAFT FRACTURES IN ADULTS
}

\author{
ABSTRACT
}

Metacarpal shaft fractures are common hand injuries which predominantly affect younger patients. There is wide variability in their treatment with no consensus on best practice. We performed a systematic review to assess the breadth and quality of available evidence supporting different treatment modalities for metacarpal shaft fractures of the finger digits in adults. A comprehensive search was conducted across multiple databases, in line with Preferred Reporting Items for Systematic Reviews and Meta-Analyses (PRISMA) guidelines. A total of 1600 records were identified; seven studies fulfilled eligibility criteria and were included. No randomised controlled trials directly comparing surgery to non-surgical treatment were found. One retrospective study compared non-surgical to surgical treatment, while six compared surgical or non-surgical treatments. Considerable heterogeneity between studies along with a high or critical risk of bias restricts direct comparison and conclusions.

There is a lack of high quality evidence to guide treatment, supporting the need for welldesigned, multi-centre trials to identify the most effective and cost-efficient treatment for metacarpal shaft fractures in adults. 
Metacarpal shaft fractures (MSF) are common injuries, accounting for $10-31 \%$ of all hand fractures. ${ }^{1-6}$ They place a significant burden on healthcare resources and society, commonly affecting young economically active patients.

Despite their prevalence, acceptable parameters of deformity vary widely in the literature ${ }^{7-9}$ and there is no consensus on the best practice management approach. Non-surgical treatment includes closed reduction, various different casting techniques and splints or free mobilisation. Surgical techniques include Kirchner wires (K-wires) fixation, intraosseous wires, interfragmentary compression screws, plates or external fixators.

Both non-surgical and surgical treatment require significant resources and a period of rehabilitation of weeks to months, during which use of the hand is restricted. Surgical treatment is perceived to be more costly due to the need for specialist resources, additional equipment and theatre use.

Whilst the majority of patients have excellent outcomes, if not appropriately treated, MSF can limit range of motion and grip strength, lead to an extensor lag from shortening, and (rarely) rotational deformity of the digit. ${ }^{10,11}$ This may impair hand function and affect ability to work and live at the preinjury level. As they predominantly affect those of working age, reduced ability to work during hand recovery may lead to substantial societal costs, increasing the cumulative morbidity of MSF. Therefore, establishing the most effective treatment for MSF will lead to optimal patient care and has the potential to provide economic value to the National Health Service.

We report the findings of a systematic review of the treatment of MSF. This review was undertaken to establish the benefits and risks of surgical and non-surgical treatments and to assess the quality and strength of evidence supporting each treatment modality. In analysing 
the available literature, we hope to highlight areas of uncertainty and identify learning points for the design of future studies.

\section{MATERIALS AND METHODS}

We developed a protocol in line with the Preferred Reporting Items for Systematic Reviews and Meta-Analyses (PRISMA) Statement ${ }^{12}$ and prospectively registered the review on PROSPERO (CRD42018106950).

\section{Eligibility criteria}

The eligibility criteria are detailed in Table 1 . We included studies if they compared any form of treatment, either surgical or non-surgical, for an acute fracture(s) of the metacarpal shaft of the finger digits in adult patients, however defined.

\section{Search strategy and study selection}

A comprehensive search strategy was compiled by an information specialist (DG) that included a comprehensive list of search terms and synonyms for the concepts; metacarpal bones, fractures and shaft/diaphysis (Supplemental Material). The following bibliographic databases were searched on $16^{\text {th }}$ September 2019: PubMed, Ovid MEDLINE, Ovid Embase, Cochrane Central Register of Controlled Trials (CENTRAL), CINAHL, Web of Science and PEDro (Supplemental Table S1). We devised a strategy specific to each database, ensuring use of the relevant subject headings where available. We screened the reference list of included studies for further eligible studies and searched the grey literature at the time of the primary search via Google Scholar. No date or language limits were applied.

Study selection is reported in a PRISMA flow diagram. Two authors (RT and DG) independently screened titles and abstracts for eligibility. Full text articles were reviewed where abstracts were unclear. Disagreements were resolved by discussion with a third 

author (AK). EndNote version X8 (Thomas Reuters, New York City, NY, USA) was used to manage search results and filter duplicate articles.

\section{Data management and risk of bias assessment}

69

Data extraction and assessment of methodological quality was performed in duplicate using a piloted data collection form (RT \& SD). Risk of bias was assessed using the Cochrane Risk of Bias Tool for Randomised Controlled Trials and quasi-random studies ${ }^{13}$ and the Risk of Bias in Non-Randomised Studies of Interventions (ROBINS-I) for comparative nonrandomised studies. ${ }^{14,15}$

\section{Data synthesis}

Data collected included information on study design, population, intervention, outcomes, including use of clinical and patient reported outcome measures (PROMs) and results. A meta-analysis was planned, if appropriate, but not performed due to study heterogeneity and risk of bias in included studies; a narrative synthesis is therefore presented.

\section{RESULTS}

The study selection process is demonstrated via a PRISMA flow diagram (Figure 1). A total of 1600 records were identified through database searches; seven studies fulfilled the eligibility criteria and were included.

Two discontinued and four ongoing trials were identified via the World Health Organisation International Clinical Trials Registry Platform (WHO ISCTRP) portal and a further six records were identified on searching the grey literature and reference lists of included studies (Supplemental Table S2).

\section{Study design characteristics}


88 There were no published randomised controlled trials (RCT) directly comparing surgical to

89 non-surgical treatment for MSF in adult patients. One observational study compared non-

90 surgical to surgical treatment. This was a retrospective, two-centre cohort study of

91 metacarpal neck and shaft fractures. ${ }^{16}$

92 Six studies made comparisons between either surgical or non-surgical treatments, as

93 summarised in Table 2. These included two RCTs, ${ }^{17,18}$ one multi-centre retrospective

94 study, ${ }^{19}$ one dual-centre retrospective study and three single-centre retrospective cohort

95 studies, as defined by the literature. $.^{20,21} 16,22-24$ Of these, three compared two forms of surgical treatment, ${ }^{19,22,23}$ and three compared non-surgical treatments. ${ }^{17,18,24}$ Two studies assessed MSF only, ${ }^{18,23}$ with the remainder being mixed population studies, which reported results for MSF as separate subgroups.

\section{Risk of bias assessment}

All studies were assessed to be at critical risk of bias in at least one domain, or serious risk of bias in two or more domains (Tables 2-4). Supplementary material detailing the quality assessment for each individual study is available on request.

As the majority of studies are retrospective, allocation of treatment may be influenced by multiple confounding factors including clinician preference, injury pattern and severity of fracture. Of the two RCTs, one used an inadequate method of randomisation (sequentiallynumbered sealed envelopes ${ }^{18}$ ) and the second did not specify the method used. ${ }^{17}$ Only one study provided a prior sample size calculation, ${ }^{18}$ therefore studies may lack the power required to detect meaningful differences between interventions.

109 Studies had variable length of follow-up, ranging from 3 weeks to 65 months, with wide inter110 participant variability within individual studies, ranging from 3 weeks to $15-65$ months, ${ }^{18,23}$ as

111 well as a disproportionate loss to follow-up between intervention groups..$^{16,19}$ 
112 Insufficient information regarding blinding of outcome measurements was provided, ${ }^{19}$ or

113 assessment of outcomes occurred at variable time points. ${ }^{16,22,23}$ Outcome measurements

114 were unblinded in all studies bar one ${ }^{24}$ and intervention groups were therefore identifiable

115 (either due to presence of surgical scars or the use of cast/splints in non-surgical

116 interventions), thus risk of bias was assessed as 'serious' for all subjectively reported

117 outcomes.

118 In some studies, there was a disparity between planned methods described and reported

119 results, thus leading to bias in selection of reported results. Furthermore, no protocols were 120 published a priori for any of the included studies, further potentiating the risk of selective 121 reporting.

122 The majority of studies did not provide sufficient information to assess bias due to deviations 123 from intended interventions ${ }^{16-19,22-24}$ or missing data. ${ }^{24}$ Therefore, bias in these domains was 124 not demonstrably measured.

\section{Participant and fracture characteristics}

126 A total of 438 participants with MSF were included in the seven studies. All studies had a 127 small sample size, mean of 63 (range 26-139).

Participants varied widely with some studies defining age restrictions while others did not. Gender was not documented in three studies, two studies had higher proportions of male participants ${ }^{19,22}$ and one contained no female participants. ${ }^{23}$

131 Eligibility criteria varied markedly between studies, particularly in definition of displacement, 132 affected digits, multiplicity of fingers fractured, inclusion criterion and indications for surgery.

133 One study defined displacement as dorsal angulation $>30^{\circ}$ or shortening $>3 \mathrm{~mm},{ }^{22}$ while two 134 did not specify minimum parameters of deformity or indications for surgery. ${ }^{19,23}$ All three comparative studies of surgical treatments excluded open fractures and two excluded highenergy/polytrauma or patients with multiple fractures. 
137 One RCT included only closed stable MSF of the fingers, defined as $<50 \%$ displacement of

138 the width of the shaft, $<40^{\circ}$ angulation and displaying an angle of $>60^{\circ}$ between the plane of 139 the fracture and the axis of the shaft, ${ }^{18}$ while the second RCT did not specify any exclusion 140 criteria, simply recruiting 100 consecutive patients. ${ }^{17}$ Information regarding inclusion 141 criterion, selection of participants, indications for treatment and choice of intervention were not provided in two studies. ${ }^{17,24}$

\section{Interventions and rehabilitation}

144 Surgical interventions, time to surgery, surgical technique and choice of metalwork varied considerably amongst studies, with some including the addition of crossed K-wires as well as intramedullary fixation ${ }^{23}$ and variability in surgical pates, including dynamic compression, locking plates or unspecified types. One study compared closed reduction and K-wire

148 fixation to open reduction and internal fixation (ORIF) using locking plates and screws. ${ }^{23}$

149 Another compared intramedullary K-wire fixation to interfragmentary screw fixation ${ }^{22}$ whilst 150 the third compared percutaneous K-wire fixation to ORIF using plate-screw fixation or 151 interfragmentary lag screws. ${ }^{19}$

152 There was a lack of consistency in mode of immobilisation, position, material used (plaster, 153 thermoplastic or other) and period of immobilisation amongst the three comparative studies 154 of non-surgical treatments (Table 2$) \cdot{ }^{17,18,24}$

\section{Outcome measures}

156 A combination of outcome measurements were used at varying time-points. Five studies

157 reported radiographic parameters, such as antero-posterior angulation, shortening or

158 presence of bridging callus. ${ }^{16,17,22-24}$ Total active motion was reported in three studies ${ }^{18,19,23}$ 159 and grip strength in three..$^{16,22,23}$

160 A PROM was reported in four of the seven studies, with the MAYO, ${ }^{22}$ QuickDASH ${ }^{16,19}$ and DASH most frequently used. ${ }^{16,22,23}$ Other clinical parameters reported included hand volume 
162

163

164

165

166

167

168

169

170

171

172

173

174

175

176

177

178

179

180

181

182

183

184

185

186

187

and finger circumference as surrogate markers of oedema, ${ }^{18}$ while post-operative rehabilitation and therapy use was only reported in one study..$^{19}$ Though return to work was recorded by Konradsen et al., it was not separately reported for MSF. ${ }^{17}$

\section{Results of included studies}

Only one study directly compared surgical to non-surgical treatment, assessing outcomes of metacarpal fractures at 2 years or more post injury. ${ }^{16}$ Though baseline demographics were similar between the groups, there was significant disparity in the number of patients per intervention, 113 treated non-surgically versus 26 surgically, as well as greater palmar angulation at presentation in the surgically treated group. No significant differences in grip strength were reported, though improved DASH scores and aesthetic outcome were noted in those managed non-surgically, along with a worse sportsDASH score. ${ }^{16}$ The reported findings suggest non-surgical treatment might be preferable to surgical fixation in the treatment of a single MSF.

Two of the three studies of surgical treatments found no evidence of any difference in either functional or PROMs between treatment groups. Biz and lacobellis found no evidence of difference when comparing intramedullary fixation to interfragmentary screw fixation at a mean follow-up of 28.4 months. ${ }^{22}$ These findings were supported by Vasilakis et al. who found no difference in functional outcomes, outpatient follow-up or hand therapy referral rates between ORIF and percutaneous pinning using K-wires. They noted that both interfragmentary screws and plate-screw fixation resulted in earlier splint removal and mobilisation compared to closed reduction and percutaneous pinning. ${ }^{19}$ Only one paper reported improved outcomes in grip strength, range of motion and DASH scores with platescrew fixation over percutaneous K-wire fixation, which they attributed to the use of lowprofile locking plates and screws that allowed for aggressive mobilisation post-surgery. ${ }^{23}$ One study reported reduced immobilisation time with ORIF (plate-screw fixation or screw fixation only) ${ }^{19}$ whilst another reported a higher incidence of malunion in those treated with 
intramedullary wire fixation over interfragmentary screw fixation. ${ }^{22}$ Given the variability in surgical interventions and lack of clearly reported indications for surgery within studies, comparisons between type of fixation and functional outcomes are not appropriate.

191 Of the comparative studies of non-surgical treatments, few reported subgroup results for MSF. Konradsen et al. described good outcomes following their "functional cast", however rotation, pain, cast inconvenience, length of time before returning to work, range of motion (ROM) and grip strength were not separately reported for MSF. ${ }^{17}$ McMahon et al. demonstrated improved ROM with immediate mobilisation and a compression glove in the first 3 weeks post-injury, though this improvement was not sustained at 4 weeks. ${ }^{18}$ Braakman concluded that near anatomical reduction of MSF resulted in reduced residual angulation at 4 weeks. ${ }^{24}$ However, these clinical improvements were not correlated with functional assessments or PROMS, therefore extrapolating these conclusions to guide patient treatment may not be appropriate.

This review highlights the paucity of high quality evidence demonstrating superiority of any one form of treatment over another for the management of MSF of the finger digits. Despite their prevalence, there is considerable variability in the management of MSF with no agreement in the literature as to acceptable parameters of deformity nor a consensus on treatment strategies. The limited studies identified lacked consistency of endpoints, surgical techniques, rehabilitation regimens and outcome measures utilised. This makes meaningful comparison difficult due to the considerable heterogeneity.

209 Only one retrospective study directly compared surgical to non-surgical treatment for MSF. ${ }^{16}$

210 As intervention and comparator groups were defined some time following injury, any

211 differences identified may be due to confounding of either patient or fracture characteristics.

212 The low follow-up rate, imbalance in numbers per intervention and variable length of follow213 up, challenges the conclusions drawn that outcomes are favourable following either form of 
214 treatment. ${ }^{16}$ There was also differential attrition in the treatment groups, which is likely due to

215 systematic differences between the two groups.

216 Despite increasing trends towards surgical fixation in current practice, no single technique

217 has been demonstrated to be superior in the treatment of MSF. Only one retrospective study reported improved outcomes with plate-screw fixation over percutaneous pinning with $\mathrm{K}$ -

219 wires. ${ }^{23}$ However, the small sample size (59 patients), significant disparity in length of follow-

220 up between groups and serious overall risk of bias impedes the use of this study in drawing

221 conclusions about the superiority of either form of treatment. A recent meta-analysis of plate

222 fixation versus percutaneous pinning for unstable metacarpal fractures concluded that whilst

223 percutaneous pinning resulted in higher motion scores, there were no differences in

224 functional scores, grip strength, radiographic parameters, time-to-union or complications. ${ }^{25}$

225 However, this review was limited by the small number of eligible studies (only four

226 comparative studies, of which only three reported total active motion and two reported

227 DASH), a lack of standard reporting and limited use of functional outcome scores or PROMs. ${ }^{25}$

Given the heterogeneity in data and inconsistency in reporting throughout the literature, there is no evidence to support any one treatment over another for MSF. Furthermore, the following inconsistencies compounded analysis of the literature:

1. There is no clear definition of the metacarpal "shaft", with the majority of studies containing a heterogeneous group of neck and shaft fractures. One suggested definition may be that described by the Arbeitsgemeinschaft für Osteosynthesefragen Foundation/Orthopaedic Trauma Association (AO/OTA) as that part of the bone between the two end segments, with the end-segment defined by "a square whose sides are the same length as the widest part of the epiphysis/metaphysis in question (Heim's system of squares)". ${ }^{26}$ However, only one study defined the shaft using this method. ${ }^{23}$ Accurate denotation of the metacarpal shaft is required to differentiate 
mixed-population studies that include subcapital/neck fractures, which most agree tolerate far greater angulation than MSF.

2. There is no consensus on definition of instability or acceptable parameters of deformity in MSF. One study defined displacement, ${ }^{22}$ while others did not specify minimum parameters of deformity or indications for surgical treatment. ${ }^{19,23}$ Diao suggested up to $10^{\circ}$ angulation was acceptable in the index and middle fingers and $20^{\circ}$ to $30^{\circ}$ in the ring and little finger, while some authors accept up to $50^{\circ}$ angulation in the little and $30^{\circ}$ to $35^{\circ}$ in the ring ringer. ${ }^{27}$ Others are more conservative accepting $60^{\circ}$ of angulation in the little finger and $45^{\circ}$ in the ring finger. ${ }^{28}$ Similarly, while some authors opine that finger metacarpals may tolerate 3 to $4 \mathrm{~mm}$ of shortening, ${ }^{29}$ sometimes more ${ }^{23,27,28}$ with minimal clinical deformity and functional loss, cadaveric studies demonstrate that every $2 \mathrm{~mm}$ of metacarpal shortening may result in as much as $8 \%$ loss of grip strength. ${ }^{30}$ The inconsistency in reporting of fracture characteristics and deformity increases the risk of selection bias when comparing treatments for MSF and highlights the uncertainties within the hand surgery community regarding acceptable parameters of deformity in MSF. Future studies should use clear definitions of deformity alongside standardised methods of assessment to allow head-to-head comparison of treatments.

3. Though angulation and shortening were assessed in the majority of studies, precise methods of measuring deformity in MSF are not described in the literature, with some remaining as vague as stating radiographs were "scanned for metacarpal angulation and shortening". ${ }^{23}$ Angulation is often measured on lateral radiographs of the hand using mid-medullary measurement, however this method has only been validated in the assessment of metacarpal neck fractures.${ }^{31}$ Furthermore, normal reference values for angulation are only documented for the ring and little finger metacarpal. ${ }^{32}$ An accurate and reliable method of measuring angulation and shortening in MSF is required to ensure consistency in assessment across studies. Furthermore, there is no clear evidence that radiographic outcomes directly correlate with function. 
Standardising radiographic assessment alongside collection of PROMs would aid our understanding of this.

4. The majority of studies did not examine rehabilitation/therapy regimens or other key variables such as the time from injury to surgery or length of immobilisation, which may also have a prognostic impact on outcomes following MSF.

5. Where cosmesis or inconvenience of treatment has been assessed, arbitrary measures selected by study authors were used. ${ }^{16,17,22}$ Patients may have widely differing views to clinicians and acceptability to patients may vary significantly from

6. There is incongruity in outcomes assessed, with studies measuring a variety of outcomes at varying time-points. All studies focused on clinical and radiographic outcomes, with no study reporting a PROM as the primary outcome of interest. The lack of standardised reporting and assessment is compounded by the fact that there is no core outcome set for trials/studies in hand surgery. Consensus on a minimum

7. Low recruitment and retention are inherent issues in studies of metacarpal fractures and have led to the termination of several RCTs, including a multi-centre RCT of dataset in future trials is required to ensure consistency in reporting and allow future meta-analysis. intramedullary wiring and conservative treatment for subcapital and shaft fractures of the little finger metacarpal. ${ }^{33}$ This limits the pool of available clinical trials and reduces the robustness of evidence available for synthesis of meaningful conclusions regarding treatments for MSF. Future studies must minimise attrition using novel techniques, remote data collection, timely, focused follow-up and reducing research burden.

8. Studies rarely examined the socioeconomic impact of time off work, lost productivity or need for additional support/care whilst undergoing treatment for MSF. There is no evaluation of cost-effectiveness of treatments for MSF, with utilisation of resources 

rarely recorded in studies. Only one study recorded length of surgery and hospital stay. ${ }^{22}$ Such evidence is required to inform healthcare allocation.

298 Our conclusions must be considered in lieu of the study limitations. Our review is limited by 299 the small number of eligible studies, which provide mostly level IV evidence. Whilst a comprehensive search strategy was devised, it is possible that relevant publications may not

301 have been identified. As with any review, reporting bias, both within individual studies and in 302 relation to published findings, limits the available data from which to pool results. This is 303 compounded by the small sample size in individual studies. Furthermore, the high risk of 304 bias and associated limitations of included studies impedes any meaningful assessment of 305 specific intervention types and associated outcomes. We recommend that future researchers 306 address the deficiencies of prior studies, so that direct comparisons can be made between 307 treatments (Table 5).

308 This review highlights the need for large, well designed randomised studies to inform current 309 practice and guide management of these common injuries. Although RCTs are difficult to 310 implement, identifying the most beneficial and cost-effective treatment for MSF will aid 311 clinicians and patients to make informed treatment choices, whilst maximising value for 312 health service providers. 


\section{$314 \quad$ Funding}

315 This work was supported by the British Society for Surgery of the Hand and the National

316 Institute for Health Research [NIHR300197]. RT is funded by a National Institute for Health

317 Research Doctoral Research Fellowship for this research project. This article presents

318 independent research funded by the National Institute for Health Research (NIHR). The

319 views expressed are those of the authors and not necessarily those of the NHS, the NIHR or

320 the Department of Health and Social Care.

321

322 Conflict of Interests

323 The authors declare that they have no conflict of interest.

324

325 Statement of Human and Animal Rights

326 This is not applicable to this systematic review article.

327

328 Statement of Informed Consent

329 This is not applicable to this systematic review article. 
1. de Jonge JJ, Kingma J, van der Lei B, Klasen HJ. Fractures of the metacarpals. A retrospective analysis of incidence and aetiology and a review of the Englishlanguage literature. Injury. 1994;25:365-369.

2. Hove LM. Fractures of the hand. Distribution and relative incidence. Scand J Plast Reconstr Surg Hand Surg. 1993, 27: 317-9.

3. Stanton JS, Dias JJ, Burke FD. Fractures of the tubular bones of the hand. J Hand

4. Nakashian MN, Pointer L, Owens BD, Wolf JM. Incidence of metacarpal fractures in

5. Chung KC, Spilson SV. The frequency and epidemiology of hand and forearm

6. Angermann P, Lohmann M. Injuries to the hand and wrist. A study of 50,272 injuries.

7. Faraj AA, Davis TR. Percutaneous intramedullary fixation of metacarpal shaft

8. Kollitz KM, Hammert WC, Vedder NB, Huang JI. Metacarpal fractures: treatment and

9. Retrouvey H, Morzycki A, Wang AMQ, Binhammer P. Are We Over Treating Hand Fractures? Current Practice of Single Metacarpal Fractures. Plast Surg (Oakv). 2018, 26: $148-53$.

10. Strauch RJ, Rosenwasser MP, Lunt JG. Metacarpal shaft fractures: the effect of shortening on the extensor tendon mechanism. J Hand Surg Am. 1998, 23: 519-23.

11. Low CK, Wong HC, Low YP, Wong HP. A cadaver study of the effects of dorsal angulation and shortening of the metacarpal shaft on the extension and flexion force ratios of the index and little fingers. J Hand Surg Br. 1995, 20: 609-13.

12. Moher D, Liberati A, Tetzlaff J, Altman DG. Preferred reporting items for systematic reviews and meta-analyses: the PRISMA statement. BMJ. 2009, 339: b2535.

13. Higgins JPT, Altman DG, Gøtzsche PC, et al. The Cochrane Collaboration's tool for assessing risk of bias in randomised trials. BMJ (Clinical research ed). 2011, 343: d5928-d.

14. Sterne JAC, Hernán MA, Reeves BC, et al. ROBINS-I: a tool for assessing risk of bias in non-randomised studies of interventions. BMJ. 2016, 355: i4919.

15. Schünemann HJ, Cuello C, AkI EA, et al. GRADE guidelines: 18. How ROBINS-I and other tools to assess risk of bias in nonrandomized studies should be used to rate the certainty of a body of evidence. J Clin Epidemiol. 2019, 111: 105-14. 
16. Westbrook AP, Davis TR, Armstrong D, Burke FD. The clinical significance of malunion of fractures of the neck and shaft of the little finger metacarpal. J Hand Surg Eur. 2008, 33: 732-9.

17. Konradsen L, Nielsen PT, Albrecht-Beste E. Functional treatment of metacarpal fractures 100 randomized cases with or without fixation. Acta orthopaedica Scandinavica. 1990, 61: 531-4.

18. McMahon PJ, Woods DA, Burge PD. Initial treatment of closed metacarpal fractures. A controlled comparison of compression glove and splintage. J Hand Surg Br. 1994, 19: 597-600.

19. Vasilakis V, Sinnott CJ, Hamade M, Hamade H, Pinsky BA. Extra-articular Metacarpal Fractures: Closed Reduction and Percutaneous Pinning Versus Open Reduction and Internal Fixation. Plast Reconstr Surg Glob Open. 2019;7(5):e2261.

20. Mathes T, Pieper D. Clarifying the distinction between case series and cohort studies in systematic reviews of comparative studies: potential impact on body of evidence and workload. BMC Medical Research Methodology. 2017, 17: 107.

21. Dekkers OM, Egger M, Altman DG, Vandenbroucke JP. Distinguishing case series from cohort studies. Ann Intern Med. 2012, 156: 37-40.

22. Biz C, lacobellis C. Comparison of percutaneous intramedullary Kirschner wire and interfragmentary screw fixation of displaced extraarticular metacarpal fractures. Acta Biomedica. 2014, 85: 252-64.

23. Dreyfuss D, Allon R, Izacson N, Hutt D. A Comparison of Locking Plates and Intramedullary Pinning for Fixation of Metacarpal Shaft Fractures. Hand (New York, NY). 2019, 14: 27-33.

24. Braakman M. Is anatomical reduction of fractures of the fourth and fifth metacarpals useful? Eur J Radiol. 1996, 22: 38-41.

25. Melamed E, Joo L, Lin E, Perretta D, Capo JT. Plate Fixation versus Percutaneous Pinning for Unstable Metacarpal Fractures: A Meta-analysis. J Hand Surg Asian Pac Vol. 2017, 22: 29-34.

26. Meinberg EG, Agel J, Roberts CS, Karam MD, Kellam JF. Fracture and Dislocation Classification Compendium-2018. J Orthop Trauma. 2018, 32 Suppl 1: S1-s170.

27. Kozin SH, Thoder JJ, Lieberman G. Operative treatment of metacarpal and phalangeal shaft fractures. J Am Acad Orthop Surg. 2000, 8: 111-21.

28. Orbay J. Intramedullary nailing of metacarpal shaft fractures. Tech. 2005, 9: 69-73.

29. Freeland $\mathrm{AE}$, Orbay JL. Extraarticular hand fractures in adults: a review of new developments. Clin Orthop. 2006, 445: 133-45.

30. Meunier MJ, Hentzen E, Ryan M, Shin AY, Lieber RL. Predicted effects of metacarpal shortening on interosseous muscle function. J Hand Surg Am. 2004, 29: 689-93.

31. Sletten IN, Nordsletten L, Hjorthaug GA, Hellund JC, Holme I, Kvernmo HD. Assessment of volar angulation and shortening in 5th metacarpal neck fractures: an 
inter- and intra-observer validity and reliability study. J Hand Surgery Eur. 2012, 38: 658-66.

32. Braakman M. Normal radiographic angulation in the 4th and 5th metacarpal: a reference guide. Eur J Radiol. 1996, 22: 38-41.

33. Rossvoll I.ClinicalTrials.gov [Internet]: National Library of Medicine (US). 2010 Nov 17 - Identifier NCT01242982, Subcapital and shaft fractures of the 5. Metacarpal, 2010. https://clinicaltrials.gov/ct2/show/NCT01242982. Updated Apr 08, 2015. Accessed Sep 16, 2019. 


\section{FIGURE LEGENDS}

415 Figure 1 PRISMA flow diagram detailing study selection 


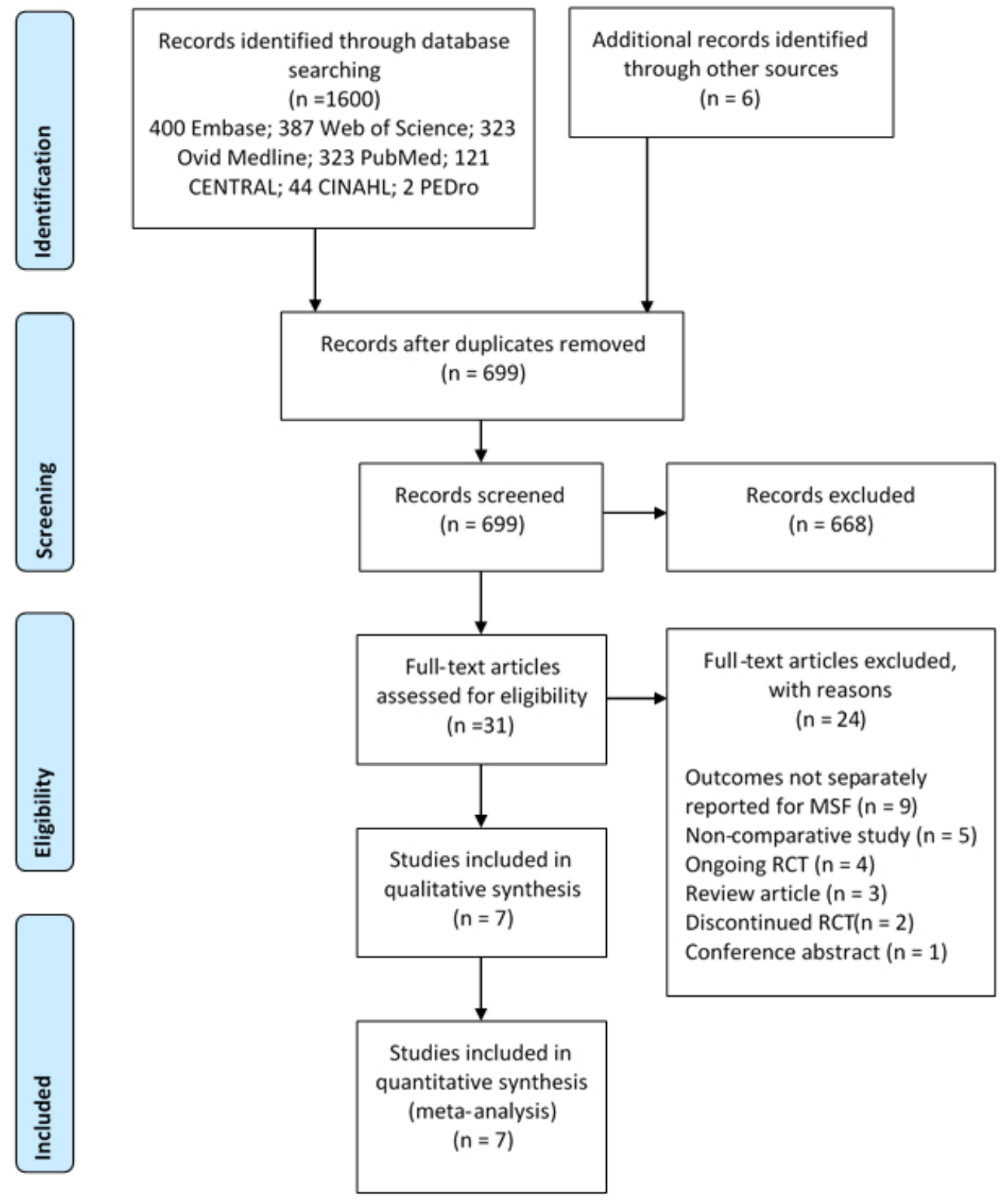

Figure 1 PRISMA flow diagram detailing study selection 
Table 1 Eligibility criteria

Inclusion criteria

\section{Types of participants}

- $\quad$ Adults, however defined, with one or more fracture(s) of the metacarpal shaft affecting the fingers (index to little)

\section{Exclusion criteria}

- Intra-articular fracture(s)

- Fracture(s) of the metacarpal neck and/or base

- Fracture(s) of the thumb metacarpal

- In studies of mixed populations (excluding adults and children) a study will be included if $>=$ $90 \%$ of the population meets the review inclusion criteria

\section{Study design}

- Randomised controlled trials

- Studies stated to be

"randomised" but for which

there is inadequate information

about sequence generation

and/or concealment of

allocation

- Controlled clinical trials

- Quasi-randomised trials, such

as those with alternate
- Cadaveric studies

- Biomechanical studies

- Case series

- Case reports

- Review articles 
allocation or allocation based on

day of the week or clinic

- Cohort studies

\section{Publication type}

- Full study reports published in peer review journals

- Separate publications of economic evaluation of the primary study

- Studies in any language
- Abstracts of completed studies, if full published report is not yet available

- Unpublished trials

- Ongoing trials/studies 
Table 2 Characteristics of included studies

\begin{tabular}{|c|c|c|c|c|c|c|c|c|}
\hline Study & Methods & $\begin{array}{l}\text { Participant } \\
\qquad \begin{array}{l}\text { s } \\
\text { n patients } \\
\text { (shaft) }\end{array}\end{array}$ & Fracture & $\begin{array}{l}\text { Intervention } \\
\text { n patients } \\
\text { (shaft) }\end{array}$ & $\begin{array}{l}\text { Comparator } \\
\text { n patients } \\
\text { (shaft) }\end{array}$ & $\begin{array}{l}\text { Follow-up } \\
\text { mean, } \\
\text { (range) }\end{array}$ & $\begin{array}{c}\text { Primary } \\
\text { outcome of } \\
\text { interest } \\
\text { (other } \\
\text { outcomes } \\
\text { assessed) }\end{array}$ & $\begin{array}{c}\text { Risk of bias } \\
\text { as per } \\
\text { Cochrane } \\
\text { assessmen } \\
\text { t tool }^{\mathrm{a}}\end{array}$ \\
\hline & annaingl th & tment & & & & & & \\
\hline $\begin{array}{l}\text { Westbrook } \\
\text { et al., } 2008\end{array}$ & $\begin{array}{l}\text { Retrospectiv } \\
\text { e cohort } \\
\text { study }\end{array}$ & 262 (139) & $\begin{array}{l}\text { Isolated } \\
\text { closed shaft or } \\
\text { neck fracture } \\
\text { of the little }\end{array}$ & $44(26)$ & $\begin{array}{l}\text { Non-surgical } \\
\text { treatment; early }\end{array}$ & $\begin{array}{l}I^{\mathrm{b}:} \\
25 \text { months } \\
(14-79)\end{array}$ & $\begin{array}{l}\text { Angulation } \\
\text { Grip strength } \\
\text { DASH }\end{array}$ & Critical \\
\hline
\end{tabular}

a Revised Cochrane risk-of-bias tool for randomised trials (Rob 2) used for randomised controlled trials. Risk of bias in non-randomised studies of interventions (ROBINS-I) tool was used for non-randomised studies.

$\mathrm{b}$ Intervention 


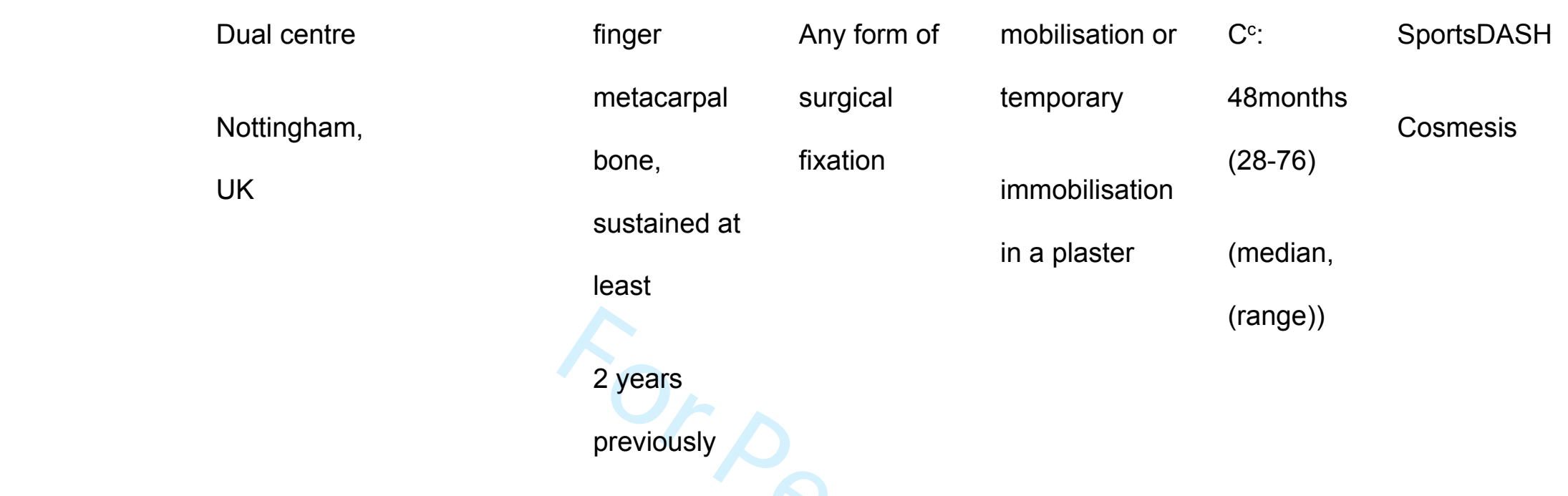

\section{Surgical v surgical treatment}

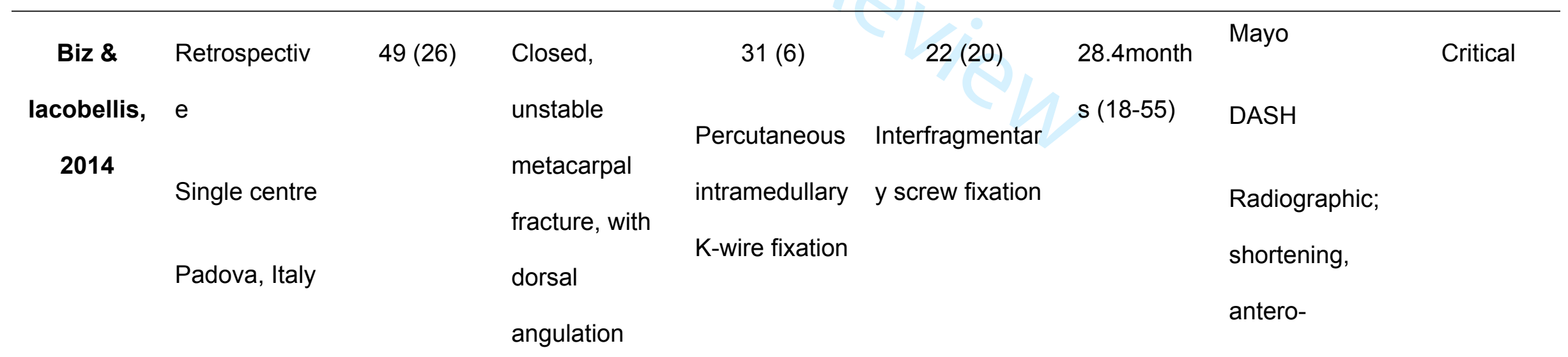

c Comparator 


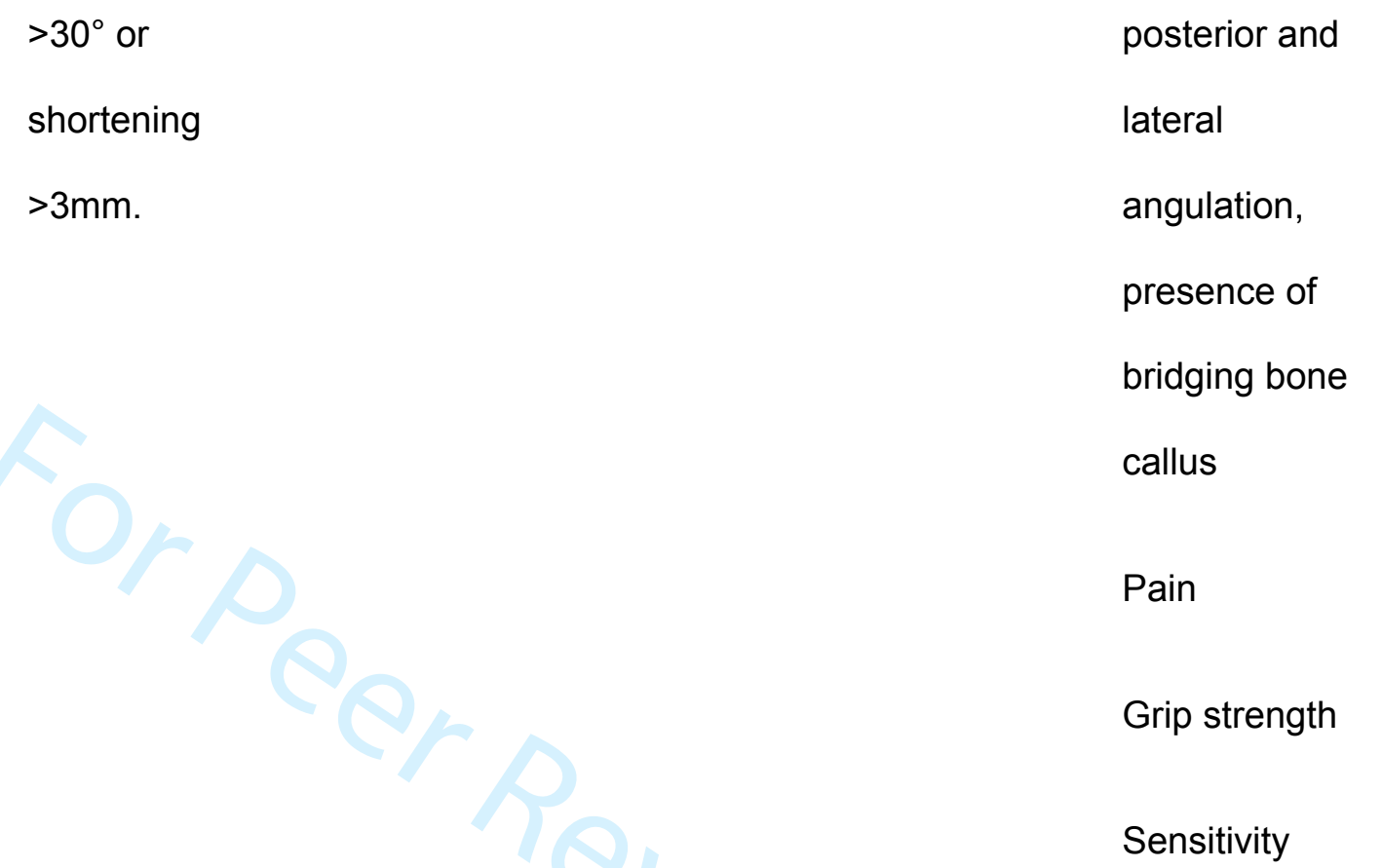

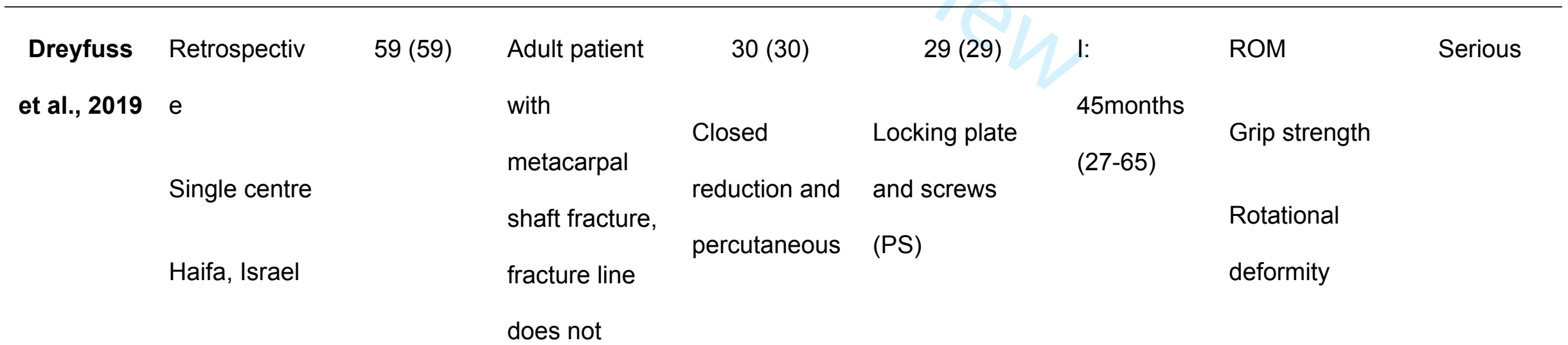




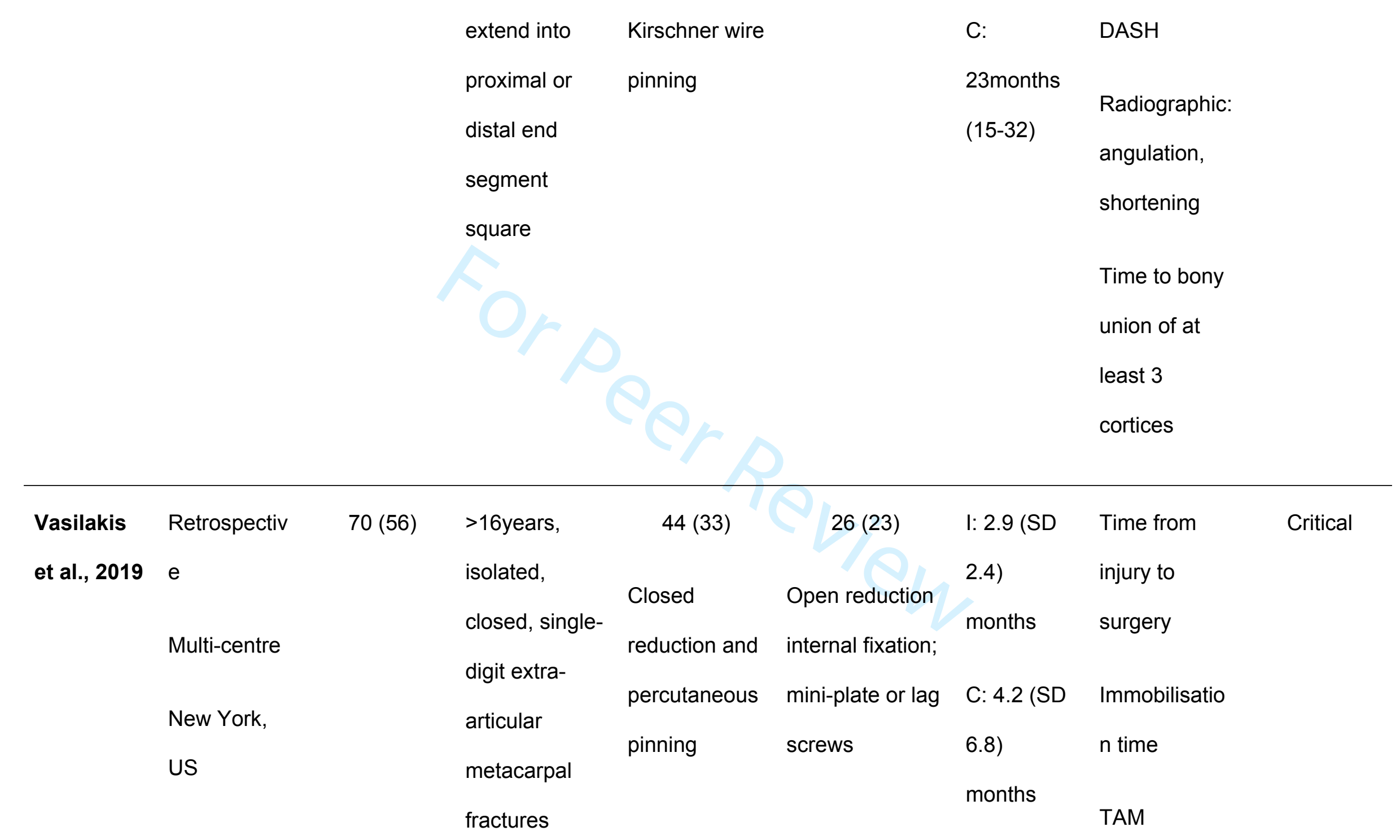




\section{Complication}

\section{Reoperation}

rate

\section{OT referral}

rates

Duration of

OT

QuickDASH

\section{Non-surgical v non-surgical}

\begin{tabular}{ll}
\hline Konradse & RCT \\
n et al., & Single centre \\
1995 & Hillerød, \\
& Denmark
\end{tabular}

$\begin{array}{cccc}100(42) & \text { Shaft or neck } & 50(22) & 50(20) \\ & \text { fracture index } & & \\ \text { to little finger } & \text { Immobilisatio } & \text { Immobilisation } \\ \text { metacarpal } & \mathrm{n} \text { in functional } & \text { in plaster cast, } \\ & \text { cast, allowing } & \text { immobilising the }\end{array}$




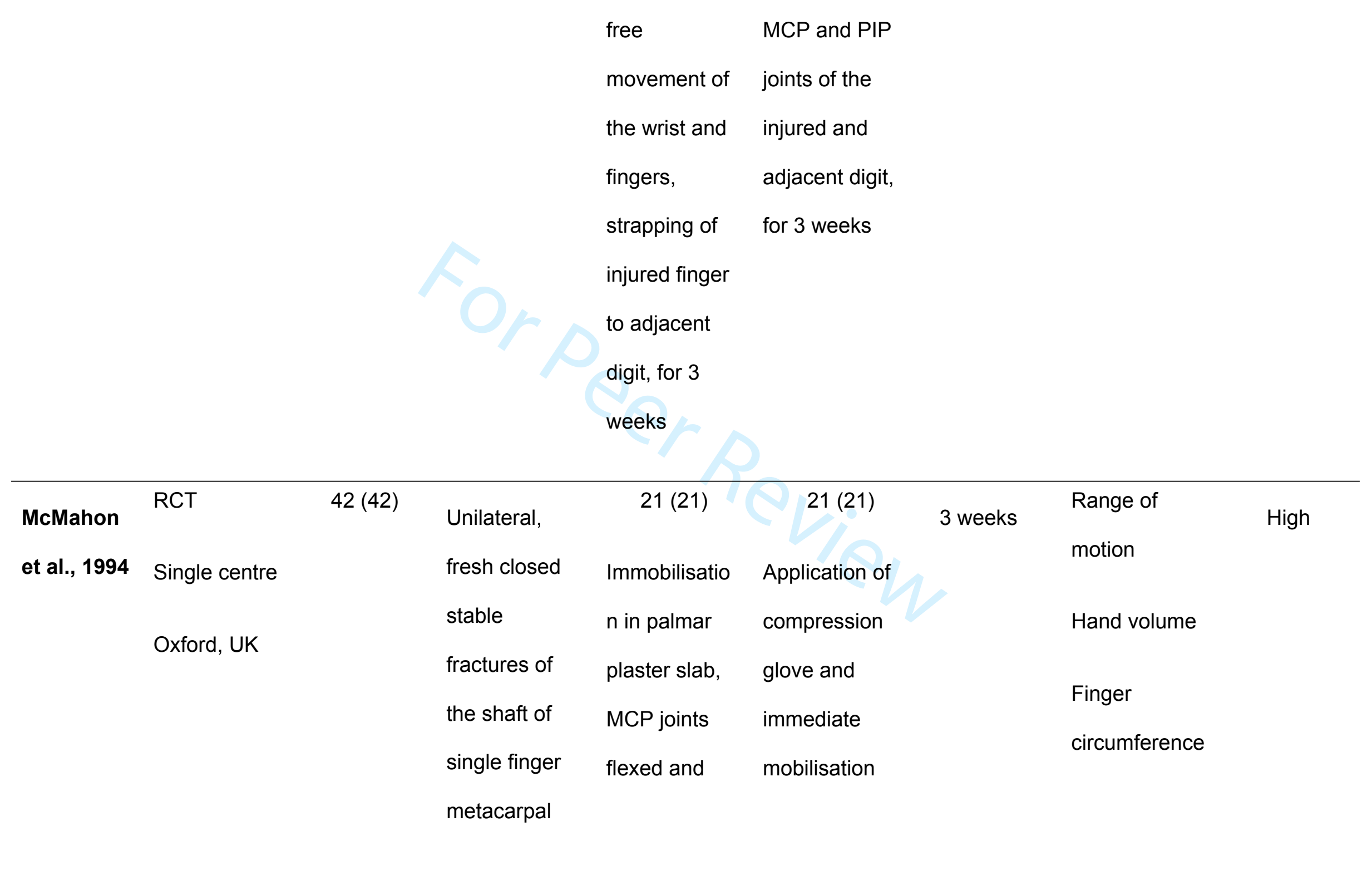


PIP joints

extended

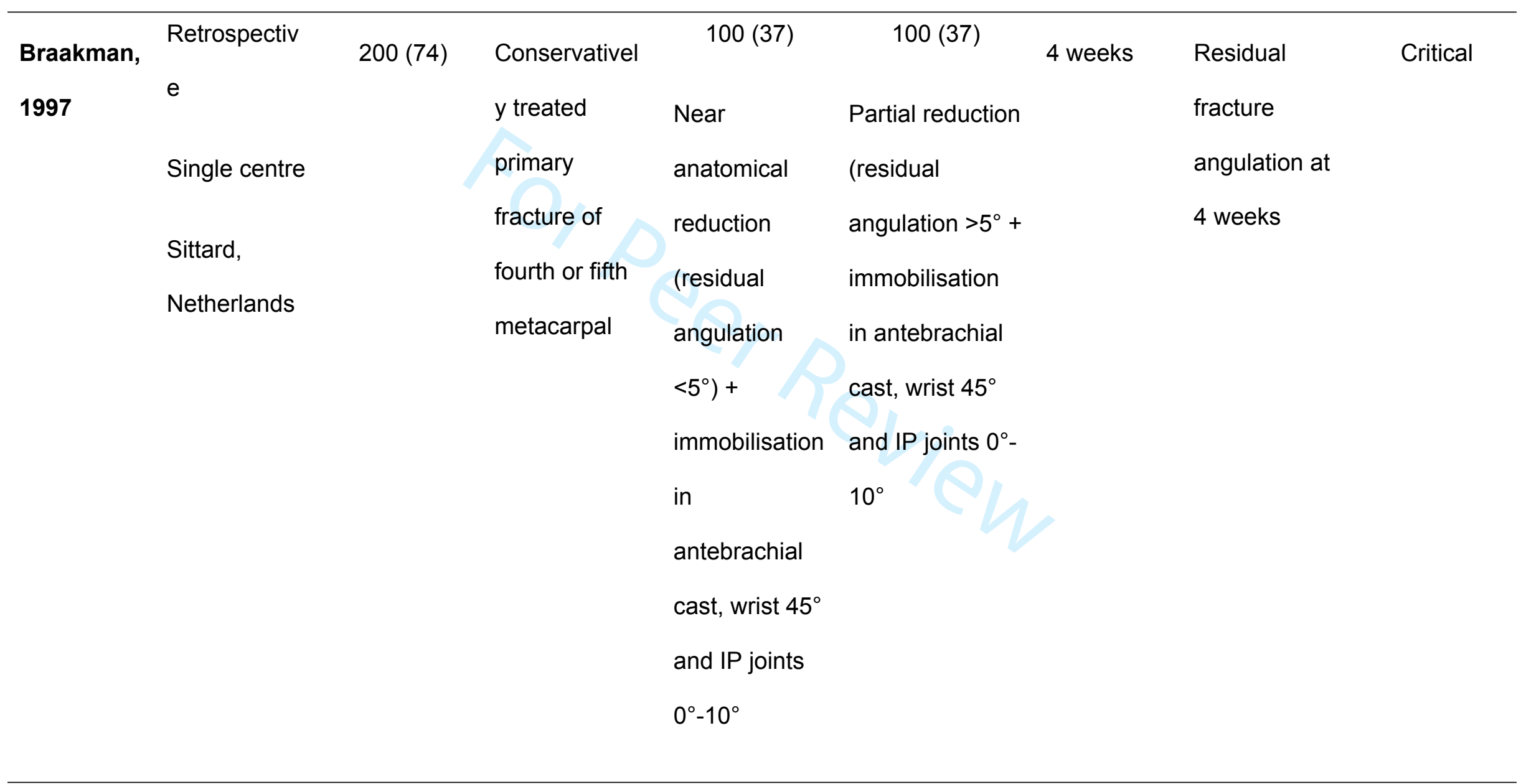


Table 3 Consolidated summary of risk of bias for non-randomised studies

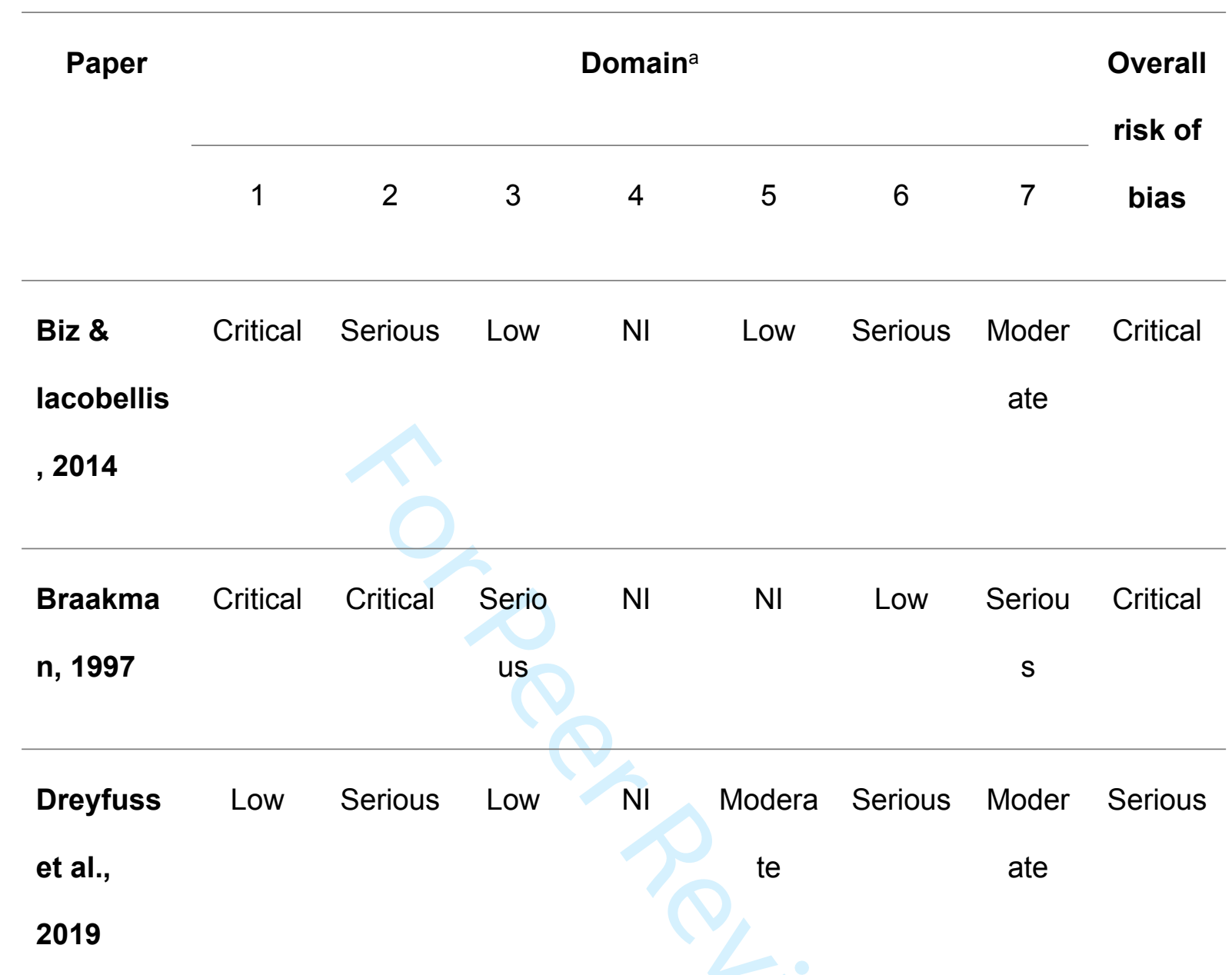

Vasilakis Critical Serious Low NI Serious NI Seriou Critical et al., $\mathrm{s}$ 2019

Westbroo Critical Serious Moder NI Critical Serious Moder Critical k et al., ate ate 2008

\footnotetext{
a Domain 1: Bias due to confounding. Domain 2: Bias in selection of participants into the study. Domain 3: Bias in classification of interventions. Domain 4: Bias due to deviations from intended interventions. Domain 5: Bias due to missing data. Domain 6: Bias in measurement of outcomes. Domain 7: Bias in selection of the reported result. $\mathrm{NI}-$ No information.
} 
Table 4 Summary of risk of bias assessment for randomised studies

\begin{tabular}{|c|c|c|c|c|c|c|}
\hline \multirow[t]{2}{*}{ Paper } & \multicolumn{5}{|c|}{ Domain $^{a}$} & \multirow{2}{*}{$\begin{array}{c}\text { Overal } \\
\text { risk of } \\
\text { bias }\end{array}$} \\
\hline & 1 & 2 & 3 & 4 & 5 & \\
\hline $\begin{array}{l}\text { Konradsen } \\
\text { et al., } 1995\end{array}$ & $\begin{array}{c}\text { Some } \\
\text { concerns }\end{array}$ & High & Low & High & Some & High \\
\hline $\begin{array}{l}\text { McMahon } \\
\text { et al., } 1994\end{array}$ & $\begin{array}{c}\text { Some } \\
\text { concerns }\end{array}$ & $\begin{array}{c}\text { Some } \\
\text { concerns }\end{array}$ & Low & High & Some & High \\
\hline
\end{tabular}

a Domain 1: Risk of bias arising from the randomisation process. Domain 2: Risk of bias due to deviations from the intended interventions (effect of assignment to intervention). Domain 3: Missing outcome data. Domain 4: Risk of bias in measurement of the outcome. Domain 5: Risk of bias in selection of the reported result. 
Table 5 Our recommended minimum dataset for future metacarpal shaft studies

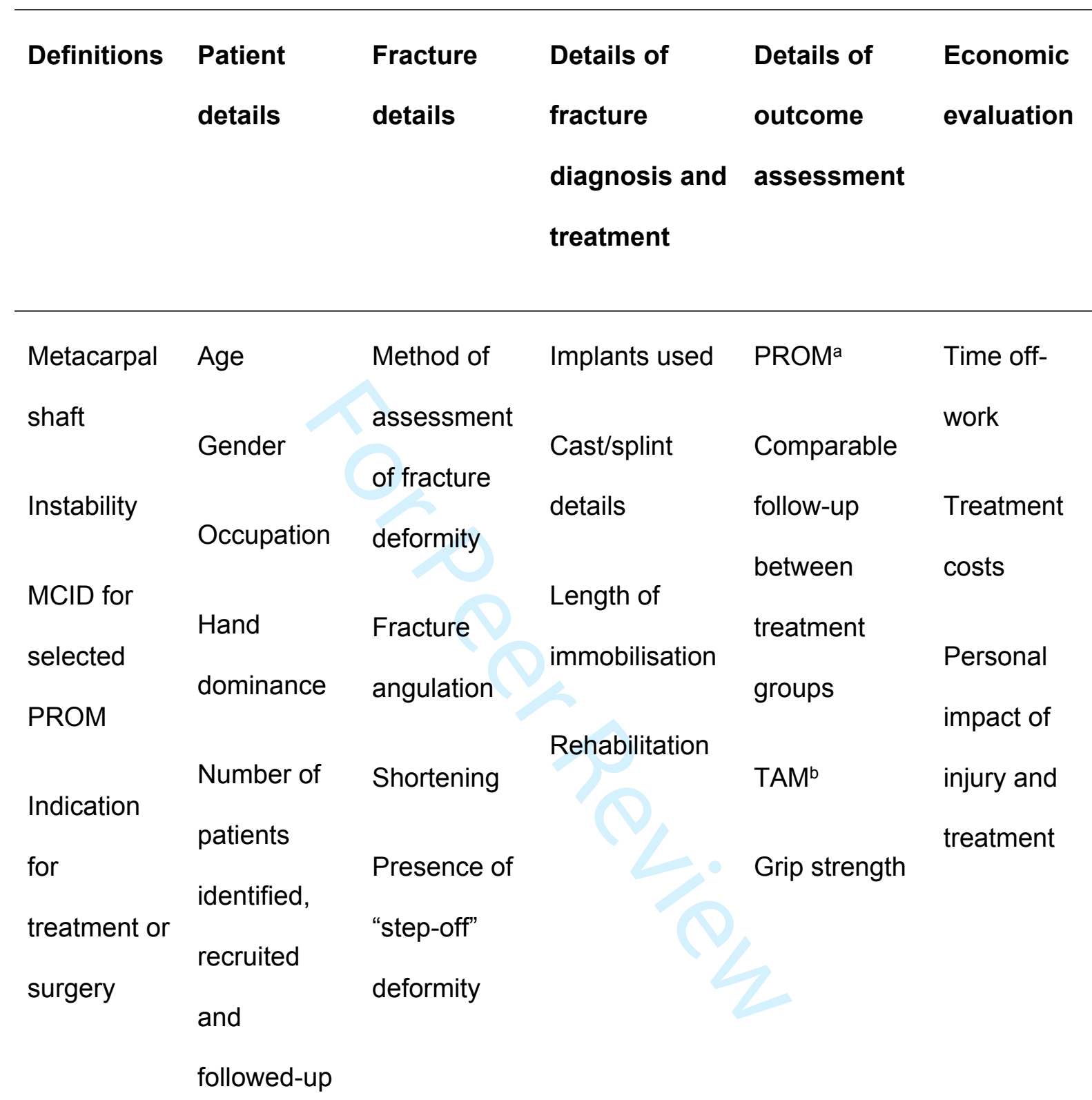

\footnotetext{
a Patient Reported Outcome Measure

b Total Active Motion
} 


\section{SUPPLEMENTAL MATERIAL}

\section{OVID Medline search strategy}

3 1. metacarpal.mp. [mp=title, abstract, original title, name of substance word, subject heading

4 word, floating sub-heading word, keyword heading word, protocol supplementary concept

5 word, rare disease supplementary concept word, unique identifier, synonyms]

6 2. metacarpals. $\mathrm{mp}$. [mp=title, abstract, original title, name of substance word, subject

7 heading word, floating sub-heading word, keyword heading word, protocol supplementary

8 concept word, rare disease supplementary concept word, unique identifier, synonyms]

9 3. transmetacarpal.mp. [mp=title, abstract, original title, name of substance word, subject

10 heading word, floating sub-heading word, keyword heading word, protocol supplementary

11 concept word, rare disease supplementary concept word, unique identifier, synonyms]

12 4. midmetacarpal.mp. [mp=title, abstract, original title, name of substance word, subject

13 heading word, floating sub-heading word, keyword heading word, protocol supplementary

14 concept word, rare disease supplementary concept word, unique identifier, synonyms]

15 5. exp Metacarpal Bones/

6. 1 or 2 or 3 or 4 or 5

17 7. fracture.mp. [mp=title, abstract, original title, name of substance word, subject heading

18 word, floating sub-heading word, keyword heading word, protocol supplementary concept

19 word, rare disease supplementary concept word, unique identifier, synonyms]

20 8. fractures.mp. [ $\mathrm{mp}=$ title, abstract, original title, name of substance word, subject heading 21 word, floating sub-heading word, keyword heading word, protocol supplementary concept 22 word, rare disease supplementary concept word, unique identifier, synonyms] 
9. fractured.mp. [mp=title, abstract, original title, name of substance word, subject heading word, floating sub-heading word, keyword heading word, protocol supplementary concept word, rare disease supplementary concept word, unique identifier, synonyms]

10. exp Fractures, Bone/

\section{7 or 8 or 9 or 10}

12. diaphysis.mp. [mp=title, abstract, original title, name of substance word, subject heading word, floating sub-heading word, keyword heading word, protocol supplementary concept word, rare disease supplementary concept word, unique identifier, synonyms]

13. diaphyses.mp. [mp=title, abstract, original title, name of substance word, subject heading word, floating sub-heading word, keyword heading word, protocol supplementary concept word, rare disease supplementary concept word, unique identifier, synonyms]

14. diaphyseal.mp. [mp=title, abstract, original title, name of substance word, subject heading word, floating sub-heading word, keyword heading word, protocol supplementary concept word, rare disease supplementary concept word, unique identifier, synonyms]

15. shaft.mp. [mp=title, abstract, original title, name of substance word, subject heading word, floating sub-heading word, keyword heading word, protocol supplementary concept word, rare disease supplementary concept word, unique identifier, synonyms]

16. shafts.mp. [mp=title, abstract, original title, name of substance word, subject heading word, floating sub-heading word, keyword heading word, protocol supplementary concept word, rare disease supplementary concept word, unique identifier, synonyms]

17. extraarticular.mp. [mp=title, abstract, original title, name of substance word, subject heading word, floating sub-heading word, keyword heading word, protocol supplementary concept word, rare disease supplementary concept word, unique identifier, synonyms] 
46 18. extra-articular.mp. [mp=title, abstract, original title, name of substance word, subject

47 heading word, floating sub-heading word, keyword heading word, protocol supplementary

48 concept word, rare disease supplementary concept word, unique identifier, synonyms]

49 19. $\exp$ DIAPHYSES/

5020.12 or 13 or 14 or 15 or 16 or 17 or 18 or 19

$51 \quad 21.6$ and 11 and 20 


\section{Supplemental Tables}

Supplemental Table S1 Summary of databases searched

\begin{tabular}{lcc} 
Database & Platform & Dates covered \\
\hline PubMed & PubMed & $1946-2019$ \\
\hline MEDLINE(R) ALL & OVID & $1946-2019$ \\
\hline EMBASE & OVID & $1974-2019$ \\
\hline $\begin{array}{l}\text { Cochrane Central Register of } \\
\text { Controlled Trials (CENTRAL) }\end{array}$ & Cochrane Library, Wiley & $1996-2019$ \\
& & \\
\hline CINAHL & EBSCOhost & $1937-2019$ \\
\hline PEDro & PEDro & $1999-2019$ \\
\hline Web of Science & Web of Science & $1900-2019$
\end{tabular}




\section{Supplemental Tables}

Supplemental Table S2 Characteristics of ongoing studies (ordered by enrolment
date)

\begin{tabular}{ll}
\hline NCT02718170 & \\
\hline $\begin{array}{l}\text { Trial name or } \\
\text { title }\end{array}$ & $\begin{array}{l}\text { Buried intramedullary K-wire fixation compared with plate and screw } \\
\text { fixation for metacarpal fractures in unstable extra-articular metacarpal } \\
\text { fractures }\end{array}$ \\
\hline
\end{tabular}

Methods Study design: parallel RCT

Random sequence generation: not reported

Allocation concealment: not reported

Masking: open-label

Participants Location: Prisma Health-Upstate, Greenville, South Carolina, USA

Target sample size (N): 110 participants

Inclusion criteria

- The patient has an unstable extra-articular metacarpal fracture that meets operative indications

- Informed consent is obtained from the patient or proxy

- Male or female who are 16 years of age or older

\section{Exclusion criteria}

- If the patients range of motion was decreased prior to injury (previous upper extremity injury, osteoarthritis, etc.)

- Pathological Fracture

- Greater than 21 days from fracture to definitive open reduction and internal fixation

- If contamination or wounds from open fractures do not permit standardized buried intramedullary fixation or plate and screw fixation

- Highly comminuted diaphyseal fractures

- Articular fractures

- Multiple fractures involving bones other than another metacarpal in the same upper extremity

- The patient had a previous upper extremity injury that has limited hand function or finger range of motion

Interventions Type of intervention

- Buried Intramedullary K-wire Fixation

Type of comparator

- Plate and Screw Fixation

\section{Outcomes Primary outcomes}


- Disability as measured by Disability of Arm, Shoulder and Hand Score

\section{Secondary outcomes}

- Total Active Motion in degrees

- Measured by goniometer

- Grip Strength

- Disability as measured by Disability of Arm, Shoulder and Hand Score

Timing of outcomes measurement: 3 months, 1 year

Starting date Main ID: NCT02718170

Date of registration: 24 March 2016

Last refreshed on: 30 May 2019

Date of $1^{\text {st }}$ enrolment: March 2015

Status: enrolling by invitation

Estimated study completion date: March 2022

\section{Contact} information

Name: John Millon, MD

Address: Prisma Health-Upstate

Telephone: not reported

Email: not reported

Affiliation: Prisma Health-Upstate

\section{ISRCTN18006607}

Trial name or Stability of unicortical versus bicortical metacarpal fracture internal title fixation trial (SUBMIT):

Methods Study design: parallel RCT

Random sequence generation: not reported

Allocation concealment: not reported

Masking: open-label

Participants Location: University Hospitals Birmingham NHS Foundation Trust, Birmingham, UK

Target sample size (N): 290

Inclusion criteria

- Aged 18 or over

- Metacarpal diaphyseal fractures that require plate fixation

- Patients undergoing anaesthesia with axillary brachial plexus regional blocks

- Acute injury (within 72 hours)

\section{Exclusion criteria}

- Under 18 years of age

- Deemed not competent to sign the consent forms 
- Pathologic fracture or a previous fracture of the same metacarpal

- Other injury to the same upper limb requiring surgery

- Major nerve injury (e.g., median, ulnar or radial)

- Multi-trauma or -fractured patient

- Revision procedure

- Pregnant patient

- Current or prior history of malignancy

\section{Interventions Type of intervention}

- Bicortical fixation (standard practice), in which both the dorsal and palmar cortices of the metacarpal are drilled though

\section{Type of comparator}

- Unicortical fixation, in which only the near cortex is drilled

\section{Outcomes Primary outcomes}

- Fracture union is assessed at 6 weeks and 6 months

\section{Secondary outcomes}

- Complication rate is monitored continually throughout study

- Fluroscopy exposure is measured during surgery

- Implant failure is measured at 6 weeks and 6 months

- Post operative stiffness is measured at 6 weeks and 6 months

- Surgical time is measured during surgery

Timing of outcomes measurement: 6 weeks, 6 months

\section{Starting date Main ID: ISRCTN18006607}

Date of registration: 19 November 2015

Last refreshed on: 22 August 2016

Date of $1^{\text {st }}$ enrolment: June 2015

Status: enrolling by invitation

Estimated study completion date: not provided

\section{Contact Name: Mr Mark Foster}

information

Address: University Hospital Birmingham NHS Foundation Trust, Plastic Surgery Department, Mindelson Way, Edgbaston, B15 2WB, UK

Telephone: not reported

Email: not reported

Affiliation: University Hospitals Birmingham NHS Foundation Trust, Birmingham and Royal Centre for Defence Medicine (UK)

Notes This trial was due to complete in April 2018 but has been extended due to poor recruitment.

\section{KCT0003863}

Trial name or Comparison of low-profile locking plate Fixation versus antegrade title Intramedullary nailing of Unstable Metacarpal Shaft Fractures 


\begin{tabular}{ll}
\hline Methods & Study design: parallel RCT \\
Random sequence generation: not reported \\
Allocation concealment: not reported \\
Masking: open-label
\end{tabular}

Interventions Type of intervention

- Low profile plate

Type of comparator

- Intramedullary nailing

\section{Outcomes Primary outcomes}

- Visual analog scale (VAS) for postoperative pain

- Disabilities of the Arm, Shoulder, and Hand (DASH) score

Secondary outcomes

- Grip Strength

Timing of outcomes measurement: 2 years

\begin{tabular}{ll}
\hline Starting date & Main ID: NCT02718170 \\
& Date of registration: 29 April 2019 \\
& Last refreshed on: not reported \\
& Date of $1^{\text {st }}$ enrolment: 14 February 2019 \\
& Status: Active, not recruiting \\
& Estimated study completion date: February 2021 \\
\hline $\begin{array}{l}\text { Contact } \\
\text { information }\end{array}$ & Name: Soo Min Cha \\
\hline
\end{tabular}


Address: Department of Orthopaedic Surgery, Chungnam National

University School of Medicine, Regional Rheumatoid and Degenerative

Arthritis Center, 640, Daesa-Dong, Jung-Gu, Daejeon, Korea

Telephone: 82-42-338-2480

Email: csm9827@hanmail.net

Affiliation: Chungnam National University Hospital

\begin{tabular}{|c|c|}
\hline \multicolumn{2}{|l|}{ NCT04001062 } \\
\hline $\begin{array}{l}\text { Trial name or } \\
\text { title }\end{array}$ & $\begin{array}{l}\text { Non-operative vs Surgical Treatment of Isolated Non-Thumb Metacarpal } \\
\text { Shaft Fractures }\end{array}$ \\
\hline \multirow[t]{4}{*}{ Methods } & Study design: parallel RCT \\
\hline & Random sequence generation: not reported \\
\hline & Allocation concealment: not reported \\
\hline & Masking: open-label \\
\hline \multirow[t]{6}{*}{ Participants } & Location: University of Missouri, Columbia, Missouri, United States \\
\hline & Target sample size $(\mathbf{N}): 100$ \\
\hline & Inclusion criteria \\
\hline & $\begin{array}{l}\text { - Adults } 18 \text { and older } \\
\text { - Native English-speaker } \\
\text { - Non-thumb isolated single metacarpal shaft closed fracture }\end{array}$ \\
\hline & Exclusion criteria \\
\hline & $\begin{array}{l}\text { - Pre-existing condition in the involved hand/wrist, hand } \\
\text { - Contracture or deformity, pre-existing stiffness } \\
\text { - Sognitive dysfunction with inability to follow rehabilitation protocol } \\
\text { - Pregacute/chronic fracture ( }>4 \text { weeks) } \\
\text { - Veteran Affairs (VA) patients }\end{array}$ \\
\hline
\end{tabular}

\section{Interventions Type of intervention}

- Surgical Fixation

For both scissoring and non-scissoring injuries surgical fixation by either pinning, dorsal plate, or lag screws will be considered. This will be determined by surgeon expertise at the time of surgical fixation. Postoperative, a volar short arm splint and immediate AROM at full range with buddy taping to adjacent digit will be indicated. Transition to removable short arm splint at week 2 after suture removal. No strengthening until clinical union.

\section{Type of comparator}

- Non-operative/conservative management For non-scissoring injuries: Placement of short-arm cast; immediate AROM with buddy taping to adjacent digit. Focus on achieving pulp-to palm distance of $<2 \mathrm{~cm}$ at first visit. Transition to removable short arm splint at week 2 (discontinue at 6 weeks or 
when non-tender). Strengthening after clinical union.

For scissoring injuries: Closed reduction in clinic/ER and placement of short-arm cast; immediate full range AROM with buddy taping to adjacent digit. Focus on achieving pulp-to palm distance of $<2 \mathrm{~cm}$ at first visit. Transition to removable short arm splint at week 2 (discontinue at 6 weeks or when non-tender). Strengthening after clinical union

\begin{tabular}{|c|c|}
\hline Outcomes & $\begin{array}{l}\text { Primary outcomes } \\
\text { - Vas Pain Score } \\
\text { - } \text { PROMIS score } \\
\text { - } \text { GASH score } \\
\text { - } \text { Extension lag } \\
\text { - Tinger range of motion } \\
\text { - Adverse events } \\
\text { Secondary outcomes } \\
\text { - None reported } \\
\text { Timing of outcomes measurement: } 6 \text { months }\end{array}$ \\
\hline Starting date & $\begin{array}{l}\text { Main ID: NCT04001062 } \\
\text { Date of registration: } 27 \text { June } 2019 \\
\text { Last refreshed on: } 25 \text { March } 2020 \\
\text { Date of } 1^{\text {st }} \text { enrolment: June } 2019 \\
\text { Status: enrolling by invitation } \\
\text { Estimated study completion date: January } 2029\end{array}$ \\
\hline $\begin{array}{l}\text { Contact } \\
\text { information }\end{array}$ & $\begin{array}{l}\text { Name: Stacee Clawson } \\
\text { Address: University of Missouri-Columbia, Missouri, United States } \\
\text { Telephone: } 573-884-9017 \\
\text { Email: clawsons@health.missouri.edu } \\
\text { Affiliation: University of Missouri-Columbia }\end{array}$ \\
\hline
\end{tabular}

